\title{
(Im)pigiamare/(im)pigiamarsi: una storia (della buonanotte) tutta italiana
}

\author{
Lucia Francalanci
}

PUBBLICATO: 12 OTTOBRE 2021

I 1 termine pigiama è collocato dal GRADIT nel Vocabolario di base dell'italiano, nella sezione delle parole di "alta disponibilità", che comprende "circa I9oo vocaboli, di uso relativamente raro nel parlare o scrivere, ma ben noti a ogni parlante perché di grande rilevanza nella vita quotidiana". Potrà stupire allora il fatto che la parola sia giunta nella nostra lingua soltanto nei primi anni del Novecento (la prima attestazione rintracciata, anteriore di due anni alla registrazione nel Dizionario moderno di Alfredo Panzini, è del I903, nel romanzo L’incubo, di Max Pemberton, pubblicato sulla rivista "Musica e Musicisti", anno 58, n. I, p. 380: T'insegneró io il modo di nascondere una lima nella tua scarpa, e potrai imparare ad entrare ed uscire dalla finestra coi pigiama di Trevor!) e che abbia una storia che arriva da lontano; pigiama viene infatti dall'inglese pyjamas o pajamas (I80o), che deriva a sua volta dal persiano e hindi pāy jāmé, propriamente 'vestito da gamba', composto di pāy 'piede, gamba' e jāmé 'vestito' (così è segnalato nel DELI, nel GDLI, nel Garzanti, nel Vocabolario Treccani online e nello Zingarelli; il GRADIT e il Sabatini-Coletti forniscono invece la forma pāy jāma). Nel DEI l'etimologia è leggermente diversa: si dà la forma indostana (denominazione della lingua urdu, nata dalla fusione di hindi e persiano) pāejjāma, dal persiano pā̄ 'piede' e gàamä 'vestimento'; anche il Devoto-Oli segnala la forma urdu pāejāma, composto di jāma 'indumento' e pāe 'piede'; nell'Etimologico sono riportate sia la forma hindi pāyjāmāa, che quella persiana pāy ḡàmā.

Il termine persiano e hindi faceva in origine riferimento a dei pantaloni larghi legati in vita. A seguito dell'occupazione britannica in territorio indiano nel XIX secolo, il pigiama fece il suo ingresso anche nella cultura occidentale: gli inglesi lo introdussero come capo di abbigliamento da notte maschile al posto della tradizionale camicia da notte. Nei moderni dizionari italiani, il termine pigiama indica un 'indumento maschile e femminile, indossato soprattutto per dormire, formato da giacca e pantaloni' (tale definizione potrebbe forse essere resa più attuale con la semplice aggiunta della maglia in alternativa alla giacca, che implica la presenza di bottoni o di una cerniera); per estensione, nel corso del Novecento ha designato anche il pigiama da spiaggia "negli anni Trenta, indumento che veniva indossato in spiaggia, costituito da giacca e pantaloni a colori vivaci e con disegni a fiori' e il pigiama palazzo 'abito da sera femminile composto da una giacca e da pantaloni molto ampi, diffuso soprattutto negli anni Sessanta'. A differenza dell'inglese, in cui il vocabolo è usato sempre al plurale, in italiano il plurale di pigiama si forma regolarmente aggiungendo la desinenza - $i$ ( $i$ pigiami), ma esistono anche attestazioni del plurale invariato (i pigiama); questa alternativa è ammessa e registrata come variante da molte grammatiche e dizionari. Ad esempio, in Serianni I989 (III. I27) si legge: "il nome pigiama può al plurale rimanere invariato oppure assumere la desinenza - $i$ : «quei pigiama di suo marito», «quei pigiami cinesi» (entrambi gli esempi da Moravia)"

Tutta italiana è invece la storia del verbo transitivo pigiamare, denominale da pigiama, usato sia nella forma più diffusa (vedremo i dati più avanti) pigiamare, con il significato di 'mettere il pigiama a qualcuno', sia nella forma riflessiva pigiamarsi, con il significato di 'mettersi il pigiama'; è possibile rintracciare anche impigiamare (e il riflessivo impigiamarsi), forma parasintetica creata con il prefisso in- (che diviene im- per assimilazione davanti a parole che cominciano con $p$ - o b-) usato con valore locativo (il nome di base ha il ruolo di oggetto localizzato, che viene posto in qualche luogo; il 
significato è "mettere $\mathrm{N}$ in/su/intorno a X": incamiciare, incappucciare, impellicciare, ecc.). Ne vediamo qualche esempio.

ieri sono rientrata alle 19,30, valerio aveva già cenato da mia madre (santa subito, davvero), così ho lavato tagliato e messo a cuocere in padella un po' di verdure (zucchina melanzana rinsecchita peperoni ammosciati patata carota scalogno). con valerio che voleva giocare (stella, ha ragione pure lui!), che poi ho pigiamato e messo a letto alle 2I. (dal forum gravidanzaonline.it, I3/2/2014)

Finalmente relax. Cena archiviata e cucina rassettata. Appendo le scarpe da running al chiodo. Veramente ci sarebbe ancora da lavare e impigiamare le creature, ma è qualcosa che si può fare con un po' più di calma. (Alessandra Iammarino, La luna è una banana e qualche volta anche un po' mela, Liberiamo.it, sez. Racconti, s.d.)

La vita da blogger è davvero piena: eventi in ogni dove, nuove conoscenze, nuovi prodotti, ispirazione continua, social e condivisione delle proprie esperienze. E tutto questo girare mi piace e mi stimola davvero tanto. Arriva pero un momento in cui la suddetta blogger deve tornare a casa, impigiamarsi (step assolutamente necessario al processo!) e concretizzare tutto ciò! (Flavia Priolo, Mens sana in casa ordinata. La nuova etichettatrice di brother, dal blog teverdeepasticcini.com, 3/1г/2016)

Oltre al significato più comune di 'mettere il pigiama', pigiamare è usato anche come verbo intransitivo nel significato di 'essere, stare in pigiama' e, estensivamente, di 'restare a casa e oziare, dedicarsi ad attività rilassanti o (in negativo) improduttive':

ho pigiamato per 15 giorni...sarà ora di far qualcosa? i biscotti fatti fuori, con il gelato, la torta di compleanno, le mandorle caramellate, i canditi d'arancia e pizza, pizza nera col salmone...ma quanto ho mangiato. mai di lunedi la dieta. troppo tempo prima della domenica di libertà! (dal blog mogliedaunavita.it, 7/I/20Io: https://mogliedaunavita.it/20ro/or/o5/cominciamo/)

Sto pigiamando in pigiama perché mi piace pigiamare. Ma forse non è una buona idea pigiamare in pigiama e vivere pigiamando, ma mi piace pigiamare quindi fottetevi mentre pigiamo perché pigiamare è la mia regola di vita. Pigiamando. (dal blog Un sussurro nella notte, 27/4/2020: https://camelia.tumblr.com/)

Oltre alle forme verbali, risultano piuttosto frequenti gli aggettivi pigiamato e impigiamato, usati anche in forma sostantivata, con il significato 'che, chi indossa il pigiama':

Il 4 gennaio serata di Lieder con il baritono Markus Werba. Mattina del dì di festa. Bambini in casa pigiamati e scatenati. Alle ir piazzateli davanti al tablet o pc: la Scala dedica loro un'oretta di divertimento e meraviglia. Una fiaba e un pezzo di quel bambino eterno che è Mozart. (I concerti del lunedi davanti al pc, "la Repubblica", 7/12/2020, p. 37)

Lo fanno già Tme e da domani Uno Mattina, che ha registrato l'uscita dal letto di numerose star televisive. Gli impigiamati hanno rifiutato di posare senza trucco: spunteranno dalle lenzuola col fondotinta. Non hanno invece opposto resistenza all'idea di mostrarsi mezzi nudi. (Massimo Gramellini, Canta il gallo, "La Stampa", 29/6/1997, p. 24)

Un'altra accezione particolare con cui occorrono gli aggettivi è quella 'che/chi indossa un indumento che ricorda o assomiglia a un pigiama' o, nel caso di oggetti inanimati, 'che ricorda o somiglia a un pigiama, fatto a foggia di pigiama'.

Anche il Dopofestival di Piero Chiambretti (che ha annunciato il ritorno in Rai, "prima o poi") è volato negli ascolti, fino a I.649.0oo telespettatori, share del 40,96\%. Una puntata in cui Piero, sempre in 
"smoking pigiamato", ha accolto Michelle Hunziker pure lei in tenuta notturna, a tu per tu con Gabriella Germani che l'ha imitata. (A Pippo si perdona tutto Parolacce, gaffes e pure la bestemmia, codacons.it, 2/3/2007)

Quest'uso risulta essere tipico soprattutto del linguaggio giornalistico; in particolare, se ne trova traccia negli articoli di moda e - curiosamente - in quelli di ambito calcistico, in cui si parla di pigiamati in riferimento a giocatori che indossano (anche occasionalmente) magliette a strisce e/o colori sbiaditi che ricordano le tinte più frequenti dei pigiami (generalmente il tono di tali articoli è scherzoso, anche se talvolta il fatto di "essere pigiamato" viene trasformato in un insulto). Riportiamo due esempi ( $\mathrm{da}$ notare il fatto che, in entrambi i casi, le forme in questione sono inserite tra virgolette):

Il tour tra gli scudetti della nobiltà calcistica tricolore, partirà sabato con la sfida dello Zini che opporrà ai biancorossi i "pigiamati" della Cremonese. [...] È da qualche stagione, difatti, che i "pigiamati" (furono denominati così nel 1913 a seguito dell'adozione dei nuovi colori sociali) progettano di far ritorno nell'olimpo del calcio nazionale [...]. (Filippo Rosace, Quando il "pigiama" era dei "grandi", Altoadige.it, $7 / 4 / 2016)$

La Gazzetta dello Sport analizza con toni duri il gioco espresso dall'Inter di Conte nel primo tempo della sfida contro il Genoa. "L'Inter aveva davanti un Genoa rinserrato in due linee chiuse formato 5-3, tra difesa e centrocampo otto giocatori a fare massa critica in trenta metri, ma nel primo tempo era lecito aspettarsi qualcosa di più e di meglio dai nerazzurri a Marassi con la maglia "pigiamata". L'Inter compassata, anzi pachidermica, del primo tempo non è venuta a capo di niente. (Inter, primo tempo horror: pachidermici e con un pigiama addosso, Calciomercato.com, 25/10/2020)

Interrogando le pagine in italiano di Google, quelle di Google libri e gli archivi dei quotidiani nazionali ("La Stampa", "la Repubblica e il "Corriere della Sera"), abbiamo provato a rintracciare le prime attestazioni sia delle forme verbali pigiamare e impigiamare (e dei rispettivi riflessivi pigiamarsi e impigiamarsi), sia degli aggettivi pigiamato e impigiamato. Sulla base dei dati raccolti abbiamo quindi provato a ipotizzare le loro trafile derivative. Tale ricostruzione si è resa necessaria soprattutto per poter comprendere il rapporto tra i verbi e gli aggettivi, che coincidono formalmente con le forme del participio passato.

La prima occorrenza rintracciata è del ı9ı6, in un articolo presente sul supplemento mensile "Noi e il mondo" del quotidiano "La Tribuna", ed è dell'aggettivo parasintetico impigiamato:

Incominciò un periodo di zeppelinfrenesia: di notte un ronzio atmosferico bastava a far accorrere sulle terrazze e sui tetti miriadi di impigiamati grossi tedeschi e di incamiciate bionde tedesche. (Romanelli, Zeppelin kommt, "Noi e il mondo", I916, p. 939)

La successiva attestazione è del I968, in un articolo della rivista "Nuovi argomenti", all'epoca diretta da Carocci, Moravia e Pasolini; anche in questo caso si tratta dell'aggettivo impigiamato, usato in contrapposizione all'aggettivo azzimato:

E lui in poltrona, azzimato, o impigiamato sul letto, nella camera più presentabile di quella sua casa insolentissima: aperta a tutte l'ore, a tutti i ficcanaso perdigiorno e snobetti in vena di visite [...]. (Dario Bellezza, La rapida ira, "Nuovi argomenti", n. Io, aprile-giugno I968, p. I44)

Loccorrenza seguente si trova in un volume del I981, Campo di battaglia di Francesco Leonetti, poeta e scrittore italiano, fondatore, insieme a Pasolini e Roversi, della rivista "Officina"; nel testo sono presenti sia il riflessivo impigiamarsi, sia l'aggettivo sostantivato l'impigiamato: 
La regola prima è infatti impigiamarsi, con varietà di scelta ma con dispositivo di elastico facile, a poterlo abbassare per i colpi d'ago a volo; e con riconoscibilità, a ogni distanza, della funzione di carne svolta qui. [...] L'impigiamato è represso, sottoposto e tagliabile. Può fare il nobile decaduto con stemma sul taschino del petto, purché in pigiama. (Francesco Leonetti, Campo di battaglia, Torino, Einaudi, I98I, p. I3)

Troviamo su Google libri attestazioni successive dell'aggettivo nel 1985 (collo impigiamato), nel I987 (zarine impigiamate), nel 1989 (ballerini-pupazzeti impigiamati), nel I99I (bambini impigiamati), e cosi via. Per quanto riguarda la forma riflessiva impigiamarsi, la seconda occorrenza si ha nel I994:

mi alzo, bacio i figli, corro in camera, mi spoglio, m'impigiamo, vado in bagno, mi lavo i denti come fa ogni americano cento per cento, m’infilo a letto. (Ivan Della Mea, Un amore di luna: vent'anni di fiabe, racconti e novelle, Bologna, Granata Press, 1994, p. in8)

Le successive sono del 2004, 2005 e 2006, tutte rintracciate in rete, su forum o blog. La prima attestazione del verbo impigiamare si ha invece nel 2000, in una rubrica presente sulla "Repubblica":

La sera cerco di tornare sempre prima delle 8 (in genere mia moglie è a casa da unoretta), si cena seduti a tavola tutti insieme e insieme si sta fino all'ora di mettere la bimba a nanna: naturalmente è lei che sceglie chi la deve impigiamare e addormentare. (Lettere a Barbara Palombelli, "la Repubblica", $27 / 4 / 2000$, p. 14)

A questa, segue un'occorrenza del 2006, presente in un forum in rete:

ore 9:30 li impigiamo di peso, li ficco sotto le coperte, arrivo alle minacce e riesco ad ottenere che tacciano (dal forum manuelfrattini.it, 27/8/2006)

In base ai dati riportati, notiamo quindi che le forme parasintetiche sono comparse precedentemente; secondo la nostra ricostruzione si forma per primo l'aggettivo parasintetico impigiamato (I9I6, I968, I98I, ecc.), con la normale possibilità di impiego anche in forma sostantivata; si forma poi il verbo pronominale riflessivo impigiamarsi (I981, I994, 2004, ecc.) e infine il corrispondente verbo transitivo impigiamare (2000, 2006, ecc.).

Per quanto riguarda invece le forme denominali non parasintetiche pigiamare/pigiamarsi/pigiamato, troviamo una prima attestazione del verbo transitivo nel 1938 :

In un negozio di camicieria in Piccadilly si leggeva: - Noi pigiamiamo il mondo -; in un negozio di materiale cartografico: - Noi mappiamo il mondo. (Maria Argenziano Maggi, Il dramma della parola, Bologna, Cappelli, I938, p. I42)

Si tratta però di un caso isolato, di un occasionalismo della moda, il cui significato non sembrerebbe essere 'noi mettiamo (materialmente) il pigiama' ma piuttosto 'noi vendiamo pigiami a tutto il mondo', 'noi diffondiamo il pigiama nel mondo'.

Troviamo la successiva occorrenza solo nel I990, nel romanzo Vi ho già tutti sognato una volta dello scrittore e giornalista italiano Maurizio Maggiani; si tratta in questo caso dell'aggettivo sostantivato pigiamato, usato nell'accezione 'che, chi indossa il pigiama':

e mi faccio schifo io, pigiamato domenicale infetto, ancora alla mia età senza una grazia, senza beltà di dentro e di fuori. (Maurizio Maggiani, Vi ho già tutti sognato una volta, Milano, Feltrinelli, 1990, p. 32) 
L'attestazione seguente è del 1995 ed è ancora dell'aggettivo pigiamato; il significato è stavolta quello di 'che ricorda o somiglia a un pigiama, fatto a foggia di pigiama':
Abulico, scarico al servizio, nullo con il rovescio, un tristissimo clone del numero uno del mondo - triste almeno come la polo beige e i bermudoni pigiamati che la Nike gli ha messo addosso - si è arreso nella semifinale «all american» del Masters in due brutti set al fosforo, alla tigna e agli ace (7) di Michelino Chang. (Sampras presuntuoso cosi Chang lo punisce, "La Stampa", 29/II/1995, p. 33)

Si rintracciano poi altre due occorrenze dell'aggettivo (in un caso sostantivato) nel 1998 , una in un articolo presente sul quotidiano "La Stampa" (free drink a tutti i pigiamati) e una in Google libri (addome pigiamato). Le successive, nei vari significati, sono del 2002, 2004, e cosi via.

La prima attestazione del verbo transitivo pigiamare, con il significato di 'mettere il pigiama a qualcuno', è del 2005:

Il compromesso è stato che li pigiamavo, fornivo loro l'acqua per la notte, e poi potevano fare quello che gli andava di fare, in camera loro, ma con la luce bassa, senza urlare, e che non mi chiamassero ancora. (Sui bambini ad alta richiesta... di nuovo, dal forum promiseland.it, 7/3/2005)

Successiva di poco è quella del verbo riflessivo pigiamarsi, che risale al 2006:

Oggi verso le I4.oo decido di partire da solo, stranamente Nadia preferisce restare a casina a vedersi un dvd... chissà perché mi domando io? Sono senza pantaloni tecnici e quindi mi pigiamo sotto i jeans, e sono senza casco integrale in assistenza rientrerà settimana prossima, ma decido che un vero biker non si deve fermare per cosi poco via un po' di dignità. (dal forum animaguzzista.com, 5/2/2006)

In base a questa ricostruzione, quindi, la trafila derivativa è la seguente: tralasciando l'esempio del I938, poiché si tratta di un hapax, di un occasionalismo, la progressione vede per prima la formazione dell'aggettivo pigiamato (I990, I995, 2002, ecc.), con la normale possibilità di impiego anche in forma sostantivata; si formano poi il verbo transitivo pigiamare e la sua forma pronominale riflessiva pigiamarsi, che risultano praticamente coevi (2005 e 2006). Compare per ultimo il verbo intransitivo pigiamare, con il significato di 'essere, stare in pigiama' e, per estensione, 'restare a casa e oziare, dedicarsi ad attività rilassanti o (in negativo) improduttive', la cui prima attestazione risale al zoro (l'esempio è quello che abbiamo già citato all'inizio).

Dunque, gli aggettivi pigiamato e impigiamato si sarebbero formati precedentemente alle forme verbali. I due verbi hanno una storia piuttosto diversa - impigiamare compare già negli anni Ottanta e ha da subito una presenza più costante, sia in rete che nei testi a stampa; pigiamare compare molto più tardi ma finisce per essere la forma predominante - ma il loro finale coincide: nel biennio 2019-2020 la presenza delle due forme verbali si fa più consistente (dal 2020 per impigiamare, dal 2019 per pigiamare), per poi decrescere nel corso del 202I (anche se i numeri rimangono più alti rispetto a prima del 2019). Questo aumento della frequenza, limitatamente al 2020, potrebbe essere legato all'epidemia, che ci ha costretto a passare molto tempo in casa, tempo che probabilmente abbiamo vissuto per la gran parte in pigiama.

In ogni caso, i dati relativi alla diffusione attuale del verbo (im)pigiamare ci rivelano che si tratta per lo più di una voce appartenente al lessico familiare, e che, probabilmente proprio per questo suo uso limitato alla comunicazione tra i membri di una famiglia o in ambienti ristretti, non viene accolta dai dizionari. Le forme verbali risultano poco diffuse in rete, nei libri e nei quotidiani, tuttavia, le poche occorrenze rintracciabili sono comunque significative della vitalità di tali vocaboli, che entrano come 
colloquialismo espressivo anche in testi scritti di tipo letterario e giornalistico e poi nel "parlato digitato" della rete.

Le pagine in italiano di Google (in data 23/9/202I) restituiscono I.350 risultati per pigiamare, 77 per pigiamarsi, I0.996 per le forme pigiamato/pigiamata/pigiamati/pigiamate (si può trattare sia degli aggettivi che delle forme del participio, difficilmente separabili nella ricerca); per quanto riguarda la forma parasintetica, le occorrenze sono 199 per impigiamare, 6I per impigiamarsi, 6.005 per impigiamato/impigiamata/impigiamati/impigiamate (vale quanto detto per pigiamato). Si nota subito la netta prevalenza del verbo pigiamare rispetto alla variante impigiamare (sommando il numero di occorrenze delle varie forme verbali, si ottengono I2.423 attestazioni per il primo verbo e 6.265 per il secondo). Lo stesso dato emerge dalla ricerca nelle pagine in italiano di Google libri: I.628 occorrenze totali per il verbo pigiamare (I risultato per pigiamare, o per pigiamarsi, 227 per pigiamato, 955 per pigiamata, 228 per pigiamati e 217 per pigiamate) e 679 per il verbo impigiamare ( 2 per impigiamare, 2 per impigiamarsi, 202 per impigiamato, 247 per impigiamata, 219 per impigiamati e 7 per impigiamate).

La presenza nella stampa nazionale è invece pressoché inesistente: nell'archivio della "Repubblica" troviamo 3 occorrenze (I di pigiamate, I di pigiamati e I di impigiamare; nell'archivio del "Corriere della Sera" i risultati sono 5 (I pigiamato, I pigiamate, I pigiamarsi, I impigiamato e I impigiamate); nell'archivio della "Stampa" troviamo 4 occorrenze (2 di pigiamati e 2 di impigiamati) e si tratta di aggettivi e sostantivi. Resta comunque indicativo il fatto che un termine tipico del parlato informale emerga, seppure timidamente, anche nello scritto giornalistico.

In rete è possibile rintracciare anche qualche attestazione del verbo spigiamare, con il significato di 'togliere il pigiama a qualcuno' (o, nel caso della forma riflessiva spigiamarsi, 'togliersi il pigiama'), ma il numero delle occorrenze è davvero esiguo: 6 risultati per la forma all'infinito, 2 per il riflessivo e i 8 per i participi passati.

Prima di concludere, vale la pena di fare un accenno anche ad altri due derivati di pigiama: pigiamista e pigiamoso. Il sostantivo maschile e femminile pigiamista è registrato soltanto dal GDLI e dal GRADIT, col significato 'chi confeziona o produce pigiami'; nel GRADIT la data di prima attestazione è il I957, ma in Google libri ne trovano esempi precedenti, che ci permettono di retrodatare il sostantivo al I931:

E le parole di nuovo conio, esotiche, impreviste? Non parliamo delle vendite «causa trasloco», [...] ma notiamo piuttosto una fabbrica di vernici che cerca «rappresentante bene introdotto», saloni che si affittano ad uso «lavoriero», sarte che cercano d'urgenza «mezzanelle», insuperabili «maglieriste»o «pigiamiste», albergatori «stagionali», liquidi «nebulizzabili» [...]. (Mercede Mùndula, Il bello stile della "quarta pagina", "L'Italia che scrive", a. XIV, I93I, p. 300)

Il GDLI lemmatizza anche il sostantivo femminile pigiamáia 'colei che confeziona pigiami, pigiamista'. Quanto alla loro diffusione, le pagine in italiano di Google restituiscono (in data 23/9/2O2I) 3.190 risultati per la forma pigiamista, 528 per pigiamisti e 1.470 per pigiamiste; soltanto 5 occorrenze di pigiamaia, 5 di pigiamaio, 3 di pigiamaie e I di pigiamai.

L'aggettivo pigiamoso non è invece accolto da nessun dizionario e risulta presente quasi esclusivamente in rete: nelle pagine in italiano di Google risultano 885 occorrenze di pigiamoso, I.29o di pigiamosa, I.790 di pigiamosi e 270 di pigiamose; solo 4 attestazioni su Google Libri, due di pigiamoso e due di pigiamosa; nessuna attestazione nei quotidiani nazionali. L'aggettivo viene usato con il significato 'che ricorda o assomiglia a un pigiama'; ne riportiamo uno dei primi esempi rintracciati: 
Ci sono altri vestiti che a casa si possono mettere e fuori no. Tutto ciò che è morbido e vecchio, per esempio è lecito: la giacca pigiamosa, il maglione oversize, la gonna ampia di seta impalpabile e perfino l'antico vestitone afgano, quello con le pietre e gli specchietti (Chiara Boni, Luigi Settembrini, Vestiti, usciamo. L'eleganza femminile e la seduzione, Milano, Mondadori, 1987, p. 65).

\section{Cita come:}

Lucia Francalanci, (Im)pigiamare/(im)pigiamarsi: una storia (della buonanotte) tutta italiana , "Italiano digitale", XVIII, 2021/3 (luglio-settembre)

DOI: $10.35948 / 2532-9006 / 2021.11627$

\section{Copyright 2021 Accademia della Crusca}

Pubblicato con licenza creative commons CC BY-NC-ND 